\title{
A quantitative study of humeral cartilage in individuals with spinal cord injury
}

\author{
H Ruckstuhl $^{1}$, J Krzycki ${ }^{2}$, N Petrou $^{2}$, B Vanwanseele $^{3}$ and E Stussi ${ }^{1}$ \\ ${ }^{1}$ Department of Mechanical and Process Engineering, Institute for Biomechanics, ETH Zurich, Switzerland; ${ }^{2}$ Swiss Paraplegic Centre, \\ Nottwil, Switzerland and ${ }^{3}$ School of Exercise and Sport Science, University of Sydney, Australia
}

\begin{abstract}
Study design: Cross-sectional design.
Objectives: In this in vivo study, we investigated the influence of different load situations on humeral cartilage thickness in individuals with paraplegia and quadriplegia.

Setting: ETH Zurich and Swiss Paraplegic Centre Nottwil, Switzerland.

Methods: A previously validated three-dimensional (3D) gradient echo MRI-sequence with selective water excitation was used. Three groups were compared: individuals with paraplegia with high shoulder demand $(n=11)$, individuals with quadriplegia with reduced load on the shoulder joint $(n=8)$ and a control group $(n=9)$. After $3 \mathrm{D}$ reconstruction, the mean cartilage thickness, local thicknesses (superior, medial, inferior) and the minimum joint space were calculated.

Results: The mean humeral cartilage thickness $(1.40 \pm 0.14 \mathrm{~mm})$ as well as the minimum joint space $(2.57 \pm 0.50 \mathrm{~mm})$ did not differ between groups. In individuals with paraplegia, the superior cartilage thickness was significantly greater than in individuals with quadriplegia $(1.47 \mathrm{~mm}$ compared to $1.28 \mathrm{~mm}, P<0.05)$. In the control group, there was a significant negative correlation between mean cartilage thickness and age $(r=-0.81)$.

Conclusion: Neither higher shoulder demand nor reduced shoulder loading leads to thinning of humeral cartilage. It is hypothesized that higher local stresses prevent local cartilage degeneration, as in normal load situations humeral cartilage thinning is occurring with age. Furthermore, joint space narrowing was only associated with inferior cartilage thickness and cartilage thickness is not related to shoulder pain.
\end{abstract}

Spinal Cord (2008) 46, 129-134; doi:10.1038/sj.sc.3102084; published online 12 June 2007

Keywords: spinal cord injuries; shoulder joint; cartilage thickness; magnetic resonance imaging

\section{Introduction}

The prevalence of shoulder pain in persons with chronic spinal cord injury (SCI) is very high (ranges from 30 to $73 \%){ }^{1}$ The most common causes of shoulder pain are overuse injuries to the rotator cuff. ${ }^{1,2}$ Describing the pathomechanism, shoulder problems in wheelchair users are thought to start with muscle imbalance, resulting in glenohumeral $(\mathrm{GH})$ instability, impingement disorders, rotator cuff tears and subsequently result in degenerative joint disease (DJD). ${ }^{3}$ However, only little is known about the incidence of DJD in the shoulder of wheelchair users, ${ }^{4-7}$ as often other symptoms are dominating. Furthermore, the impact of loading on degenerative changes of the shoulder is controversial discussed. ${ }^{6,7}$

Correspondence: H Ruckstuhl, Department of Mechanical and Process Engineering, Institute for Biomechanics, ETH Zurich, HCI E451, 8093 Zurich, Switzerland.

E-mail: hruckstuhl@ethz.ch

Received 29 September 2006; revised 28 February 2007; accepted 22 April 2007; published online 12 June 2007
DJD is also known as osteoarthrosis (OA), ${ }^{8}$ which include alterations of the articular cartilage (for example, thinning, lesions) and the underlying subchondral bone (for example, sclerosis, cyst and osteophytes formations). To prevent further progression of $\mathrm{OA}$, early detection of structural damage to the hyaline cartilage is necessary. ${ }^{9,10}$ Two common measurement methods are used to determine cartilage degeneration - either X-ray radiography (for qualitative analysis, that is, joint space narrowing (JSN)) or magnetic resonance imaging (MRI) (for quantitative investigations, for example, cartilage thickness). Radiographic $\mathrm{X}$-ray only measures changes in the cartilage indirectly and is prone to measurement errors due to incorrect positioning of the joint. ${ }^{11}$ Furthermore, radiography JSN seems to be a relatively late manifestation of $\mathrm{GH}-\mathrm{OA} .{ }^{12} \mathrm{MRI}$ can visualize cartilage directly and detect minor cartilage changes. After a strenuous training program, Kiviranta et al. ${ }^{13}$ observed a breakdown of articular cartilage due to abnormally high loading. In contrast, an earlier study by the same group found an increase in cartilage thickness after a moderate 
training program. ${ }^{14}$ Previous studies in spinal cord injured subjects have detected early changes in the knee joint cartilage due to the restricted movement and unloading. ${ }^{15,16}$ To the best knowledge of the authors, there is only one study investigating quantitative changes of cartilage morphology of the humeral head in SCI individuals. Although the joint is loaded more frequently, no change of humeral cartilage thickness of patients with paraplegia after one year postinjury was observed. ${ }^{17}$

As shown in the knee joint, quantitative MR imaging is a powerful method with high reproducibility to evaluate morphological changes of cartilage (for example, thickness, volume) in healthy volunteers. ${ }^{18}$ Furthermore, validation has been performed for the shoulder joint. ${ }^{19}$ Particular sequences with high-resolution images can be used to accurately determine cartilage morphology in joints as the shoulder joint, where highly curved and thin cartilage layers are present. After recording the images, it is essential to reconstruct the cartilage using three-dimensional (3D) digital post-processing techniques. ${ }^{20}$ Thus, objective results independent of section location and orientation are obtained. The first step during $3 \mathrm{D}$ reconstruction is to segment the cartilage, that is, to assign appropriate image points to anatomic structures such as the cartilage. ${ }^{20}$ After segmentation, cartilage thickness is accurately computed using normal vectors to the bone-cartilage interface - as shown in the knee joint. ${ }^{21}$

To summarize, no study has investigated the influence of different load situations on mean and local humeral cartilage thickness in individuals with SCI. Therefore, the specific objective of this study is to determine humeral cartilage thickness in individuals with paraplegia and quadriplegia, compared to a control group of volunteers using quantitative MRI. It is hypothesized that (i) in individuals with paraplegia, local cartilage degeneration due to high-load situations and (ii) in individuals with quadriplegia, global cartilage loss due to reduced loading are observed - in analogy to knee cartilage studies. ${ }^{13,15,16}$

\section{Materials and methods}

\section{Patients and volunteers}

This cross-sectional study investigated the influence of (i) overuse and (ii) reduced loading of the shoulder joint on humeral head cartilage in chronic SCI individuals. Three groups were studied. Group 1, individuals with paraplegia (range T1-L1) with daily arm- and shoulder-activity $(n=11)$; group 2, individuals with quadriplegia (range C3-C6) with little stress on their shoulders $(n=8)$ and group 3 , a control group of volunteers with no history of chronic shoulder pain $(n=9)$. All individuals with paraplegia were using manual wheelchairs and transferring independently. The shoulderand arm-function of individuals with quadriplegia was highly restricted making independent transfers impossible; they needed help for activities of daily living. Of the eight individuals with quadriplegia, four were consistently using electric wheelchairs, three also used manual wheelchairs on even ground indoors and one patient used only a manual wheelchair.

All subjects had to fulfill following criteria:

- Age: 60 years or younger, to exclude age-related GH degeneration. ${ }^{22}$

- Gender: male, as there is evidence of gender-related difference in cartilage thickness. ${ }^{23}$

In addition, the patient groups (group 1 and 2) were characterized as follows:

- Only patients with chronic SCI were included in this study, that is, more than 1 year post-injury. ${ }^{1}$ Results from unloaded knee joints indicate that cartilage thinning might be expected as early as 1 year post-injury. ${ }^{15,16}$ For the paraplegic group, only patients injured more than 5 years were included, as degenerative changes seem not to occur at an early stage of overuse. ${ }^{6}$

- All patients had traumatic, motoric complete SCI.

Subjects were recruited in two protocols: (i) a letter stating the purpose of this study was sent to all potential patients of the database of the Swiss Paraplegic Centre Nottwil. These patients had to fulfill the inclusion criteria mentioned above and had to be willing to participate in this study and (ii) inpatients from the Swiss Paraplegic Centre Nottwil, who fulfilled the inclusion criteria, were informed about the study and asked whether they are interested in participating.

We investigated only shoulders, which had not had any previous surgery and were on the patients' dominant side (right vs left handed). In case of surgery, we analyzed the nondominant shoulder. Mean age and standard deviation for group 1, 2 and 3 were $45 \pm 8$ years (range 32-59), $47 \pm 9$ years

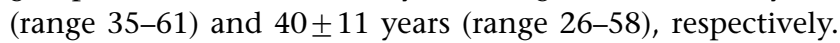
Mean post-injury age and standard deviation for group 1 and 2 were $20 \pm 10$ years (range $11-42$ ) and $15 \pm 7$ years (range $1-$ 25), respectively. Mean body mass index and standard deviation for group 1, 2 and 3 were $24.7 \pm 4.3$ (range 16.932.1 ), $22.1 \pm 1.4$ (range 20.5-24.7) and $24.9 \pm 2.4$ (range 21.628.4), respectively. All participants were informed about the experimental protocol and signed a consent approved by the local ethics committee of Lucerne, Switzerland.

Magnetic resonance imaging. The shoulder joint of all subjects were analyzed at the Swiss Paraplegic Centre Nottwil, Switzerland. The instrument used was a $1.5 \mathrm{~T}$ MR scanner (Magnetom Symphony, Siemens, Erlangen, Germany) with a shoulder array coil. A coronal data set was obtained using an image orientation perpendicular to the glenoid cavity (Figure 1). A previously validated $3 \mathrm{D}$ gradient echo sequence (FLASH) with selective water excitation ${ }^{19}$ $\left(\mathrm{TR}=30 \mathrm{~ms} ; \mathrm{TE}=10.7 \mathrm{~ms} ; \mathrm{FA}=25^{\circ}\right.$; number of averages $\left.=2\right)$ was used. High-resolution images were obtained with an in-plane resolution of $0.273 \times 0.273 \mathrm{~mm} \quad(\mathrm{FOV}=140 \mathrm{~mm}$; matrix: $512 \times 512$ pixels) and a slice thickness of $1.5 \mathrm{~mm}$. Time of acquisition was $19 \mathrm{~min} 14 \mathrm{~s}$. Manual shimming around the head of the humerus and the glenoid cavity was performed. 




Figure 1 Coronal magnetic resonance image from the glenohumeral joint. The cartilage is shown bright, the bone black. Humeral cartilage is highlighted with an arrow. Cartilage is divided into three sectors: superior, medial and inferior.

\section{Digital postprocessing}

Segmentation of the MR images was performed by one person, using a B-spline curve algorithm with manually set control points. With this method, higher interobserver precision is obtained compared to manual segmentation. ${ }^{24}$ The $2 \mathrm{D}$ spline curves were converted into a $3 \mathrm{D}$ triangular mesh. Cartilage thickness was calculated using the normal distance respective to the bone-cartilage interface, independent of the original slice orientation. In each subjects, the number of slices to segment was calculated by a fixed code based on the size of the humeral head. This method assured that only clear images from the same part of the humeral head in each individual were segmented. After analyzing images of all patients, it was calculated that only slices ranging from 42 to $68 \%$ of the whole humeral head in anterior-posterior direction should be used for segmentation.

For regional evaluation, the reconstructed cartilage was divided into three sectors of equal length (from cranial to caudal) - superior, medial and inferior (Figure 1).

The minimum joint space was calculated as the shortest distance from the bone-cartilage border (tidemark) of the humeral head to the bone-cartilage border of the glenoid in normal direction relative to the bone-cartilage interface of the humeral head.

\section{Questionnaire}

Shoulder pain was defined as positive, if subjects reported shoulder pain during or after high-load situations, during arm movements or at rest.

\section{Statistics}

The statistics was performed using the statistical software SPSS. To evaluate the statistical significance of cartilage thickness (mean, superior, medial and inferior) and minimum joint space differences between the groups, univariate analysis of variance was used. Tukey test (post hoc) was applied to determine statistical significance of observed differences. We used the Pearson's correlation (i) to analyze the influence of age on cartilage thickness and (ii) to determine the correlation of cartilage thickness and minimum joint space. The association between cartilage thickness and shoulder pain was statistically analyzed by using an independent $t$-Test. The significance level for all tests was set to $P<0.05$.

The reproducibility of the segmentation was investigated by determination of the coefficient of variation $(\mathrm{CV} \%=$ s.d. divided by the mean $\times 100$ ) from three replicated segmentations in three subjects. These three subjects were chosen such that one person from each group was represented and cartilage thickness with minimum, medium and maximum values out of the whole investigation were included in this subgroup.

\section{Results}

There was no significant difference in age between groups. Data for the mean, superior, medial and inferior humeral cartilage thickness, as well as for the minimum joint space, are listed in Table 1. There was no significant difference in mean cartilage thickness between groups. The superior cartilage of individuals with paraplegia was significantly thicker than that of individuals with quadriplegia $(P=0.045)$. The superior cartilage thickness of individuals with paraplegia was thicker compared to control group, but did not reach statistical significance $(P=0.065)$.

In the control group, the cartilage thickness decreased with age (see regression line in Figure 2). Pearson's correlation coefficients for mean, superior, medial and inferior thickness were $-0.81,-0.77,-0.89$ and -0.71 , respectively. These correlations were all significant. For the two patient groups, no statistically significant correlation between cartilage thickness and the patient's age was found.

No difference in minimum joint space between the groups was observed. The correlation coefficient of cartilage thickness and minimum joint space was always positive, but only the correlation between the inferior thickness and the minimum joint space was significant (Table 2).

Eight out of 11 individuals with paraplegia (73\%) and 3 out of 8 individuals with quadriplegia (38\%) reported shoulder pain. For the two patient groups (group 1 and 2), no difference of mean cartilage thickness of persons with and without shoulder pain could be found.

The CV\% of segmentation of the humeral cartilage for the mean value in three subjects had an averaged value of $1 \%$ and was much smaller than the intersubject variability. For the minimum joint space, coefficient of variance had an average value of 3.5\% (Table 3 and s.d. in Table 1).

\section{Discussion}

The mean humeral cartilage thickness did not differ between individuals with paraplegia and quadriplegia and controls. 
Table 1 Mean, superior, medial and inferior cartilage thickness and minimum joint space in individuals with paraplegia (group 1) and quadriplegia (group 2) and the control group (group 3)

\begin{tabular}{lcccccc}
\hline & Group & Mean & Median & s.d. & Minimum & Maximum \\
\hline Mean th. & 1 & 1.45 & 1.42 & 0.17 & 1.26 & 1.74 \\
& 2 & 1.40 & 1.35 & 0.12 & 1.30 & 1.63 \\
Superior th. & 1 & $1.47^{\mathrm{a}}$ & 1.39 & 0.22 & 1.21 & 1.87 \\
& 2 & 1.28 & 1.25 & 0.16 & 1.13 & 1.60 \\
Medial th. & 3 & 1.28 & 1.30 & 0.10 & 1.11 & 1.42 \\
& 1 & 1.75 & 1.78 & 0.25 & 1.33 & 2.20 \\
Inferior th. & 2 & 1.72 & 1.65 & 0.19 & 1.50 & 2.13 \\
& 3 & 1.60 & 1.57 & 0.22 & 1.33 & 2.01 \\
& 2 & 1.38 & 1.35 & 0.13 & 1.16 & 1.64 \\
Min. JS & 3 & 1.51 & 1.57 & 0.17 & 1.22 & 1.67 \\
& 1 & 2.66 & 2.80 & 0.43 & 1.86 & 3.32 \\
& 2 & 2.62 & 2.61 & 0.74 & 1.58 & 3.96 \\
& 3 & 2.42 & 2.51 & 0.29 & 1.94 & 2.75 \\
\hline
\end{tabular}

Abbreviations: JS, joint space; th., thickness.

Units in $\mathrm{mm}$.

${ }^{a}$ The superior cartilage of individuals with paraplegia was significantly thicker $(P<0.05)$ than that of individuals with quadriplegia.

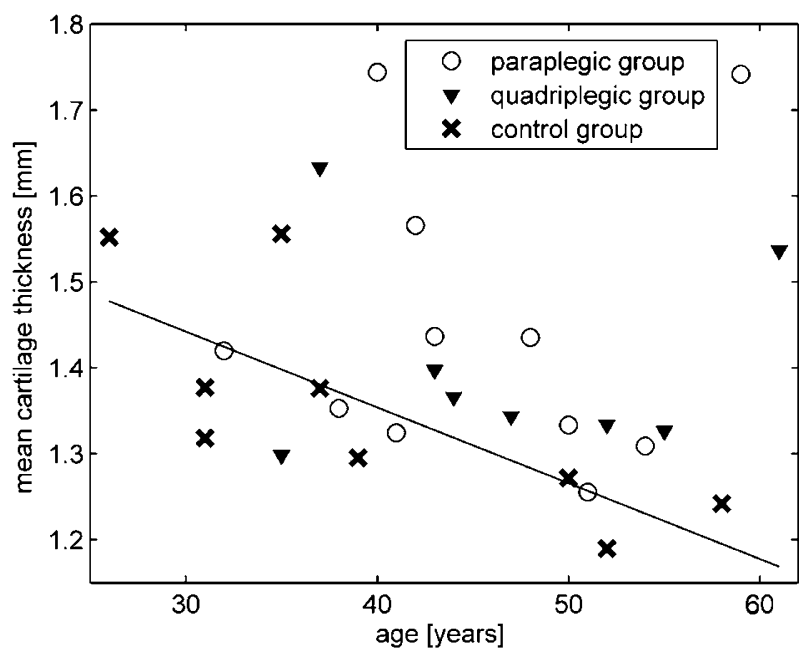

Figure 2 Mean humeral cartilage thickness distribution dependent on age. For the control group, the regression line visualizes the thickness reduction with age $(r=-0.81)$.

Furthermore, neither local thinning of humeral cartilage in individuals with paraplegia nor any global thinning in individuals with quadriplegia has been observed. That is to say that neither higher shoulder demand nor reduced shoulder loading leads to humeral cartilage thinning.

Differences between groups were found in local thicknesses. Individuals with paraplegia had thicker cartilage at the superior part of the humeral head compared to individuals with quadriplegia. In contrast, individuals with quadriplegia had thicker cartilage at the inferior part of the humeral head compared to individuals with paraplegia, although this was not found to be statistically significant. To discuss these results, it is necessary to extrapolate the progression of cartilage changes since the time of accident, that is, since the persons are wheelchair users. For that, the
Table 2 Correlation of different cartilage thicknesses and minimum joint space

\begin{tabular}{lcccc}
\hline & Mean th. & Superior th. & Medial th. & Inferior th. \\
\hline $\begin{array}{l}\text { Pearson's correlation } \\
\text { coefficient }\end{array}$ & $r=0.37$ & $r=0.27$ & $r=0.20$ & $r=0.47^{\mathrm{a}}$ \\
\hline
\end{tabular}

Abbreviation: th., thickness.

${ }^{\mathrm{a}}$ Significant correlation $(P<0.05)$

Table 3 Segmentation reproducibility in three subjects of mean cartilage thickness and minimum joint space $(C V \%=$ s.d. divided by the mean $\times 100$ )

\begin{tabular}{lcccccccc}
\hline & \multicolumn{3}{c}{ Mean cartilage thickness } & & \multicolumn{3}{c}{ Minimum joint space } \\
\cline { 2 - 3 } \cline { 7 - 9 } Subjects & Mean $(\mathrm{mm})$ & CV\% & s.d. $(\mathrm{mm})$ & & $\min (\mathrm{mm})$ & CV\% & s.d. $(\mathrm{mm})$ \\
\hline 1 & 1.74 & 1.10 & 0.02 & & 3.32 & 0.75 & 0.03 \\
2 & 1.33 & 0.97 & 0.01 & & 2.38 & 7.82 & 0.17 \\
3 & 1.19 & 0.99 & 0.01 & & 2.56 & 1.82 & 0.05 \\
\hline
\end{tabular}

Abbreviation: CV\%, coefficient of variation.

Subject 1 belonged to group 1, subject 2 to group 2 and subject 3 to group 3 .

age of the patients at time of accident as well as the assumed cartilage thickness progression in the control group were used. Thereafter, the changes of cartilage thickness since the accident to the present were considered graphically. It was found that superior cartilage thickness in individuals with paraplegia and inferior cartilage thickness in individuals with quadriplegia were maintained. It is hypothesized that higher local loads on the humeral head lead to reduced degeneration of the humeral cartilage. Individuals with paraplegia support their body weight in many situations. In this situation, the stress on the superior part of the humeral head is increased as the humerus moves upwards and impacts structures between the humeral head and the acromion. In individuals with quadriplegia with only little shoulder muscular activity, the stress on the inferior part of the humeral head may be increased as the arm is hanging down. It should be noted that the $P$-value of superior cartilage thickness in individuals with paraplegia compared to individuals with quadriplegia was just 0.045 .

In the control group, humeral cartilage loss with age occurred before the age of 60 , although, in a previous study, degenerative changes of the GH-joint were found to be present after the age of $60 .^{22}$ Moreover, Petersson ${ }^{22}$ did not detect humeral cartilage thickness changes with time, although he investigated shoulder joints with an average age of 68 years (range 18-92). In a further study as well, no change of cartilage thickness with age (range 25-75 years) was found. ${ }^{25}$ However, these studies measured only at specific sites without any 3D reconstruction of the cartilage, which could explain the discrepancy. In contrast to the age-related cartilage thickness of the control group, the same observations could not be made in the para- and quadriplegic groups. The described altered function of their shoulder may protect humeral cartilage from degeneration.

A significant correlation between minimum joint space and inferior thickness was found, that is, a small joint space is associated to thin cartilage thicknesses at the inferior 
part of the humeral head. Although significant, the Pearson's correlation coefficient with $r=0.47$ represents a low correlation. However, minimum joint space did not correlate with mean cartilage thickness. Therefore, it is speculated that JSN from radiographs might not give information about the whole humeral cartilage morphology. Moreover, the extent of cartilage changes in plain-film radiography is often underestimated. ${ }^{12}$ Due to different methods to measure the joint space and to defining JSN and the fact that radiographs are projected (two dimensional) pictures, it is not easy to compare results from radiographic studies with the present work. In the present study, no difference of minimum joint space between groups was found. These results support findings in crutch-walking patients $(n=10)$, where neither degenerative changes nor GH-JSN were detected. ${ }^{26}$ Patients with higher levels of shoulder activity might not be predisposed to develop GH-JSN. However, in another study of patients who used crutches or manual wheelchairs for daily locomotion, GH-JSN was diagnosed in 26\% (7 of 27 patients) of the investigated patients. ${ }^{7}$

We found that shoulder pain was not related to humeral cartilage thickness. There are two reasons to explain this observation: (i) cartilage is not innervated and (ii) shoulder problems in wheelchair users is thought to start with muscle imbalance, resulting in $\mathrm{GH}$ instability, impingement disorders, rotator cuff tears and subsequently result in DJD. ${ }^{3}$ In this proposed pathomechanism, other structures than the cartilage and bone might be first injured.

There was very good reproducibility of the segmentation of the humeral cartilage (average CV\% of 1\%). These measurement errors are comparable to those previously reported in the knee, ${ }^{20}$ but are much smaller than those in another shoulder study. ${ }^{17}$ The reasons for the high reproducibility compared to the shoulder study of Vanwanseele et al. ${ }^{17}$ may be that repeated measurements were not taken into account and that only a part of the humeral head was segmented. The coefficient of variance for the minimum joint space was worse than the one for the humeral cartilage, as only one thickness determined the result, and not an average value.

Limitations of this study are as follows: (i) just a small number of subjects are included, which can permit only pilot conclusions; (ii) the differences found were of low significance probably due to the small number of subjects and age differences between groups (the control group was, although not significantly, younger than the patients group); (iii) stresses on the shoulder joint in individuals with quadriplegia are different from person to person due to their own motoric ability; (iv) conclusions are only valid for men as only male subjects were investigated.

To summarize:

- Neither local thinning of humeral cartilage in individuals with paraplegia nor any global thinning in individuals with quadriplegia has been observed. This means that neither higher shoulder demand nor reduced shoulder loading leads to humeral cartilage thinning.

- It is hypothesized that higher local stresses on the shoulder joint result in reduced local cartilage degenera- tion, that is, it is assumed that cartilage thinning with age is locally reduced where there is stress.

- In the normal load situation of the control group, humeral cartilage did negatively correlate with age.

- JSN was only associated with inferior cartilage thickness.

- Humeral cartilage thickness was not related to shoulder pain.

\section{Acknowledgements}

We thank all subjects participating in this study and to the Swiss Paraplegic Centre, Swiss Paraplegics Foundation and Swiss Paraplegic Research for their collaboration and support. We also thank Mark Masteron (Calgary) for his help in correcting the English grammar, and the statistical consulting service from ETH Zurich, Switzerland.

\section{References}

1 Dyson-Hudson TA, Kirshblum SC. Shoulder pain in chronic spinal cord injury, Part I: epidemiology, etiology, and pathomechanics. J Spinal Cord Med 2004; 27: 4-17.

2 Hastings J, Goldstein B. Paraplegia and the shoulder. Phys Med Rehabil Clin N Am 2004; 15: 699-718.

3 Lee TQ, McMahon PJ. Shoulder biomechanics and muscle plasticity: implications in spinal cord injury. Clin Orthop Relat Res 2002; 403 S: S26-S36.

4 Ballinger DA, Rintala DH, Hart KA. The relation of shoulder pain and range-of-motion problems to functional limitations, disability, and perceived health of men with spinal cord injury: a multifaceted longitudinal study. Arch Phys Med Rehabil 2000; 81: 1575-1581.

5 Bayley JC, Cochran TP, Sledge CB. The weight-bearing shoulder. The impingement syndrome in paraplegics. J Bone Joint Surg Am 1987; 69: 676-678.

6 Lal S. Premature degenerative shoulder changes in spinal cord injury patients. Spinal Cord 1998; 36: 186-189.

7 Wylie EJ, Chakera TM. Degenerative joint abnormalities in patients with paraplegia of duration greater than 20 years. Paraplegia 1988; 26: 101-106.

8 Mankin HJ, Brandt KD, Shulman LE. Workshop on etiopathogenesis of osteoarthritis: proceedings and recommendations. J Rheumatol 1986; 13: 1130-1160.

9 Raynauld JP. Quantitative magnetic resonance imaging of articular cartilage in knee osteoarthritis. Curr Opin Rheumatol 2003; 15: 647-650.

10 Siebold R, Lichtenberg S, Habermeyer P. Combination of microfracture and periostal-flap for the treatment of focal full thickness articular cartilage lesions of the shoulder: a prospective study. Knee Surg Sports Traumatol Arthrosc 2003; 11: 183-189.

11 Buckland-Wright JC, Macfarlane DG, Williams SA, Ward RJ. Accuracy and precision of joint space width measurements in standard and macroradiographs of osteoarthritic knees. Ann Rheum Dis 1995; 54: 872-880.

12 Kerr R, Resnick D, Pineda C, Haghighi P. Osteoarthritis of the glenohumeral joint: a radiologic-pathologic study. AJR Am J Roentgenol 1985; 144: 967-972.

13 Kiviranta I, Tammi M, Jurvelin J, Arokoski J, Saamanen AM, Helminen HJ. Articular cartilage thickness and glycosaminoglycan distribution in the canine knee joint after strenuous running exercise. Clin Orthop Relat Res 1992; 283: 302-308.

14 Kiviranta I, Tammi M, Jurvelin J, Saamanen AM, Helminen HJ. Moderate running exercise augments glycosaminoglycans and thickness of articular cartilage in the knee joint of young beagle dogs. J Orthop Res 1988; 6: 188-195.

15 Vanwanseele B, Eckstein F, Knecht H, Stussi E, Spaepen A. Knee cartilage of spinal cord-injured patients displays progressive 
thinning in the absence of normal joint loading and movement. Arthritis Rheum 2002; 46: 2073-2078.

16 Vanwanseele B, Eckstein F, Knecht H, Spaepen A, Stussi E. Longitudinal analysis of cartilage atrophy in the knees of patients with spinal cord injury. Arthritis Rheum 2003; 48: 3377-3381.

17 Vanwanseele B, Eckstein F, Hadwighorst H, Knecht H, Spaepen A, Stussi E. In vivo precision of quantitative shoulder cartilage measurements, and changes after spinal cord injury. Magn Reson Med 2004; 51: 1026-1030.

18 Eckstein F, Heudorfer L, Faber SC, Burgkart R, Englmeier KH, Reiser M. Long-term and resegmentation precision of quantitative cartilage MR imaging (qMRI). Osteoarthritis Cartilage 2002; 10: 922-928.

19 Graichen H, Jakob J, von Eisenhart-Rothe R, Englmeier KH, Reiser $\mathrm{M}$, Eckstein F. Validation of cartilage volume and thickness measurements in the human shoulder with quantitative magnetic resonance imaging. Osteoarthritis Cartilage 2003; 11: $475-482$.

20 Eckstein F, Reiser M, Englmeier KH, Putz R. In vivo morphometry and functional analysis of human articular cartilage with quantitative magnetic resonance imaging-from image to data, from data to theory. Anat Embryol (Berl) 2001; 203: 147-173.

21 Cohen ZA, McCarthy DM, Kwak SD, Legrand P, Fogarasi F, Ciaccio EJ et al. Knee cartilage topography, thickness, and contact areas from MRI: in-vitro calibration and in-vivo measurements. Osteoarthritis Cartilage 1999; 7: 95-109.

22 Petersson CJ. Degeneration of the gleno-humeral joint. An anatomical study. Acta Orthop Scand 1983; 54: 277-283.

23 Hudelmaier M, Glaser C, Hohe J, Englmeier KH, Reiser M, Putz R et al. Age-related changes in the morphology and deformational behavior of knee joint cartilage. Arthritis Rheum 2001; 44: 2556-2561.

24 Stammberger T, Eckstein F, Michaelis M, Englmeier KH, Reiser M. Interobserver reproducibility of quantitative cartilage measurements: comparison of B-spline snakes and manual segmentation. Magn Reson Imaging 1999; 17: 1033-1042.

25 Meachim G. Effect of age on the thickness of adult articular cartilage at he shoulder joint. Ann Rheum Dis 1971; 30: 43-46.

26 Wing PC, Tredwell SJ. The weightbearing shoulder. Paraplegia 1983; 21: 107-113. 